American Medical Journal 2 (1): 32-39, 2011

ISSN 1949-0070

(C) 2011 Science Publications

\title{
The Outcome of Conservative Treatment of Closed Fracture Shaft Humerus in Adult Patients
}

\author{
Muzahim, M. Taha \\ Department of Orthopaedic Surgery, \\ Tikrit University, College of Medicine, P.O. Box, 45, Tikrit, Iraq
}

\begin{abstract}
Problem statement: Fractures of the humeral shaft are commonly encountered by the orthopaedic surgeons; accounting for approximately 3\% of all fractures. There is a wide array of good options for their treatment and controversy over the best methods for many situations. Appropriate nonoperative and operative treatment of patients with humeral shaft fractures, however, requires an understanding of humeral anatomy, the fracture pattern and the patient's activity level and expectations. Although good techniques of osteosynthesis are available, the aim of this article is to emphasize on the benefit and good outcome of conservative treatment for properly selected cases to decrease the cost and avoid the complications of surgery. Approach: During the period from Jan 2008 to Jun. 2009 seventy-eight fractures of humeral shaft were treated at Orthopaedic Department in the Tikrit Teaching hospital. 20 fractures considered suitable for the study. The patients treated conservatively by using the ' $U$ ' shaped coaptation slab and the patients evaluated both clinically and radiologically every two weeks. If there is much pain or any degree of malalignment, we shift to POP cast. Then we follow the patient clinically and radiologically every 2-4 weeks and until the fracture had united and the limb functions were restored. The outcome of treatment was assessed by specific parameters which include alignment, rate of union and limb functions. Results: This study showed that the initial deformities of angulation were considerably reduced by the use of $U$ slab and the POP cast which act as a dynamic rather than a static splint, correcting angulation to less than $30^{\circ}$ in coronal plane and less than $20^{\circ}$ in sagital plane. Manipulation of the fracture was not required and did affect neither the rate of union nor the final position, as the cast appeared to be capable of correcting angulation deformities. Perfect anatomical reduction was found not to be essential for satisfactory limb function, which was present with virus angulation and posterior bowing. The incidence of delayed union compares favorably with other reported series, although the definition of delayed union is variable. Conclusion: In fracture shaft of humerus, neither rigid immobilization nor perfect alignment are of great importance for final outcome, so conservative treatment is one of the most effective methods of treatment and the operative treatment can lead to adverse effect on the outcome in case of bad judgment and should be limited as much as possible to specific indications.
\end{abstract}

Key words: Humeral shaft fractures, modalities of treatment, conservative treatment, orthopaedic surgeons, operative treatment, angulation deformities

\section{INTRODUCTION}

Fractures of the humeral shaft are commonly encountered by the orthopaedic surgeons; accounting for approximately $3 \%$ of all fractures (Christensen, 1967).Treatment of these injuries continues to evolve as advances are made in both nonoperative and operative management. There is a wide array of good options for their treatment and controversy over the best methods for many situations (Chapman, 2003). Most humeral shaft fractures can be managed nonoperatively with anticipated good to excellent results. Appropriate nonoperative and operative treatment of patients with humeral shaft fractures, however, requires an understanding of humeral anatomy, the fracture pattern and the patient's activity level and expectations.

The goals of humeral shaft fracture management are to establish union with an acceptable humeral alignment and restore the patients to their prior level of function. Many methods have been described for the treatment of humeral shaft fractures (Epps and Grant, 1991).Both patient and fracture characteristics (patient age, presence of associated injuries, soft-tissue status and fracture pattern) need to be considered to select the appropriate treatment option. 
The closed treatment methods available include:

- Hanging arm cast

- Coaptation or U-shaped brachial splint

- Velpeau dressing

- Abduction humeral splint/shoulder spica cast

- Skeletal traction

- Functional brace

Although good to excellent results have been reported using each of these different treatment modalities, functional fracture bracing has become the most common treatment for closed humeral shaft fractures (Ward et al., 1992).

The hanging arm cast: The hanging arm cast uses dependency traction provided by the weight of the cast to effect fracture reduction. Therefore, for this technique to be effective, the patient must remain upright or semi-erect at all times. The hanging arm cast may be the definitive fracture treatment or can be exchanged for a functional fracture brace. A concern with use of the hanging arm cast is fracture distraction resulting in delayed union. The indications for use of the hanging arm cast include displaced midshaft humeral shaft fractures with shortening, particularly those fractures with an oblique or spiral pattern.

Treatment with the hanging arm cast requires meticulous attention to detail. The cast should be lightweight and applied with the elbow at $90^{\circ}$ and the forearm in neutral rotation (Fig. 1). The cast should extend at least $2 \mathrm{~cm}$ proximal to the fracture.

Three plaster or wire loops are applied at the distal forearm in dorsal, neutral and volar positions; a stockinette is passed through one of these loops and around the patient's neck. Apex anterior angulation is corrected by shortening the sling; apex posterior angulation is corrected by lengthening the sling; apex medial angulation is corrected by using the volar loop and apex lateral angulation is corrected by using the dorsal loop (Fig. 2).

Coaptation splint: The U-shaped coaptation splint with collar and cuff is indicated for the acute treatment of humeral shaft fractures with minimal shortening. A carefully molded plaster slab is placed around the medial and lateral aspects of the arm, extending around the elbow and over the deltoid and acromion (Fig. 3).

The forearm is suspended by a collar and cuff. The splint should hang free of the body. The patient is instructed in range of motion exercises of the shoulder, elbow, wrist and hand.

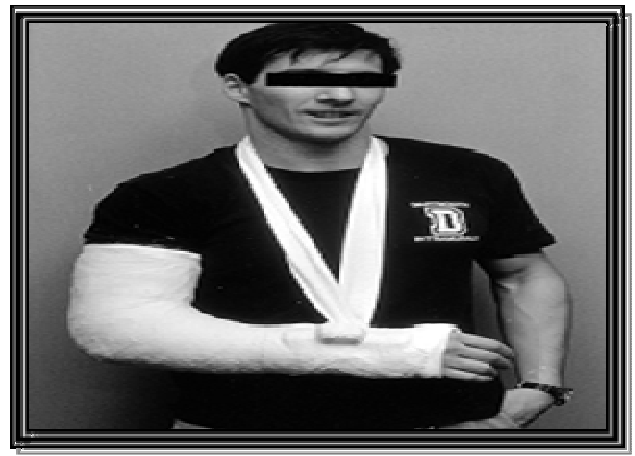

Fig. 1: The hanging arm cast is applied with the elbow 90 flexion and the forearm in neutral rotation

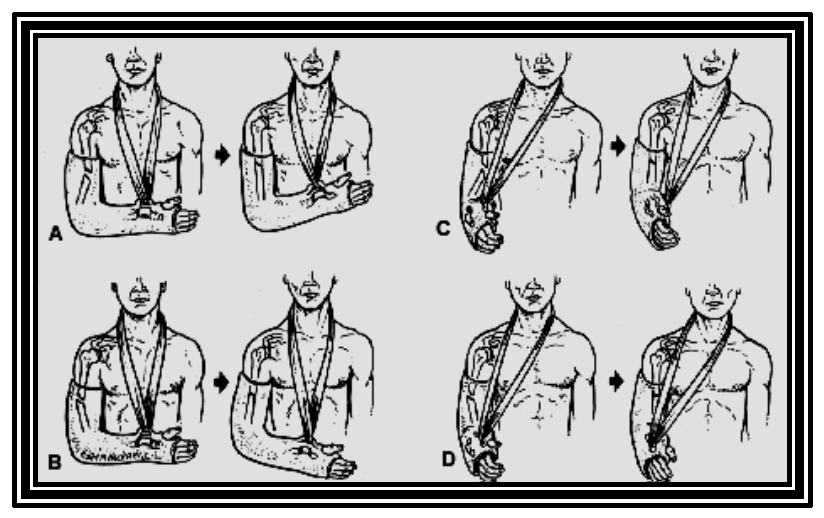

Fig. 2: (A) With use of the hanging cast, apex anterior angulation is corrected by shortening the sling; (B) Apex posterior angulation is corrected by lengthening the sling; (C) Apex medial angulation is corrected by using the volar loop; (D) Apex lateral angulation is corrected by using the dorsal loop

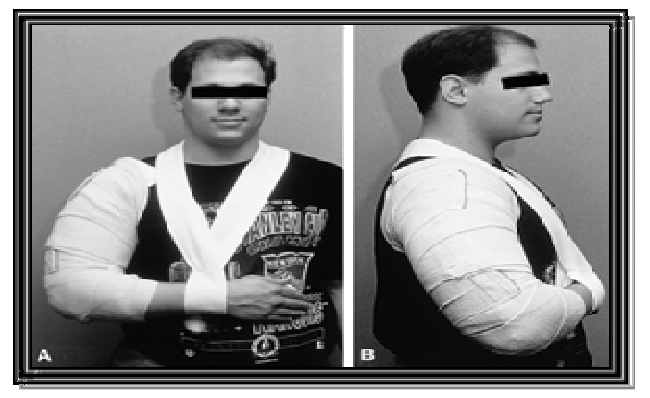

Fig. 3: U-shaped splint

Similar to the hanging arm cast, the coaptation splint is frequently exchanged for a functional cast brace 1-2 weeks after injury as the patient's pain permits (Hunter, 1982) 


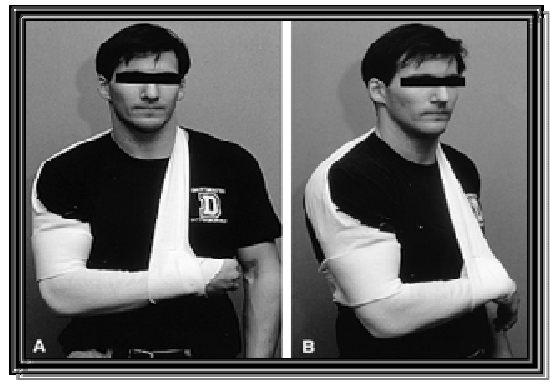

Fig. 4: A Velpeau shoulder dressing can be from a single piece of stockinette
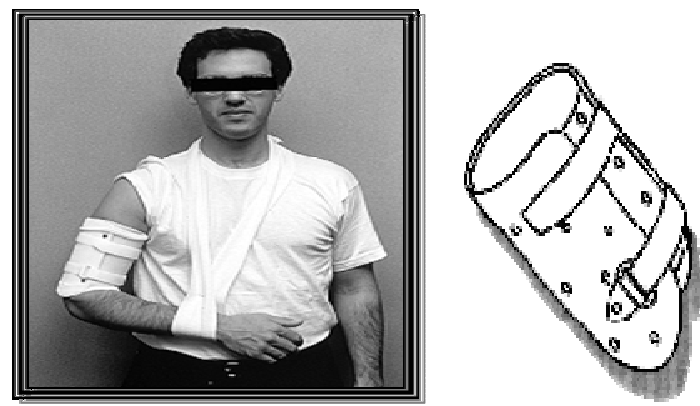

Fig. 5: A functional brace consists of an anterior shell (contoured for the biceps tendon distally) and a posterior shell, held together with Velcro straps

Thoracobrachial immobilization: A stockinette Velpeau shoulder dressing was used for immobilization of the shoulder girdle. This over-the-shoulder device is inexpensive, comfortable and easily applied (Fig. 4). This device is most useful in nondisplaced or minimally displaced fractures in children or the elderly who are unable to tolerate other methods of management.

Shoulder spica cast: The indications for use of a shoulder spica cast are unclear. The primary indications may be when closed reduction of the fracture requires significant abduction and external rotation of the upper extremity. However, when this uncommon situation occurs, operative management is frequently performed.

Skeletal traction: Skeletal traction is rarely indicated for the treatment of closed or open humeral shaft fractures. The historical indications for use of skeletal traction are now considered indications for operative intervention. When indicated, skeletal traction is applied through a transolecranon Kirschner wire or Steinmann pin. The pin should be inserted from medial to lateral to minimize the risk of ulnar nerve injury (Terry Canal, 2003).

Functional bracing: The humeral functional brace was first described by Sarmiento et al. (1977). A functional brace is an orthosis that affects fracture reduction through soft-tissue compression. Use of this device maximizes shoulder and elbow motion. This brace initially was custom made and designed as a wraparound sleeve. However, current braces are prefabricated and consist of an anterior shell (contoured for the biceps tendon distally) and a posterior shell (Fig. 5). These shells are circularized with Velcro straps, which can be tightened as swelling decreases.

Contraindications to use of the functional brace include:

- Massive soft-tissue injury or bone loss

- An unreliable or uncooperative patient

- An inability to obtain or maintain acceptable fracture alignment (Naver and Aalberg, 1986)

The humeral fracture brace can be applied acutely or 1-2 weeks after application of a hanging arm cast or coaptation splint. The brace is worn for a minimum of 8 weeks post fracture (Sarmiento et al., 1977; Naver and Aalberg, 1986).

\section{Complications:}

Radial nerve injury: Up to $18 \%$ of humeral shaft fractures have an associated radial nerve injury. Most nerve injuries represent a neurapraxia or axonotmesis; 90\% will resolve in 3-4 months (Pollock et al., 1981).

Vascular injury: Although uncommon, injury or laceration of the brachial artery can be associated with fractures of the humeral shaft. Fractures complicated by vascular injury constitute an orthopaedic emergency. Stabilization of the fracture is mandatory to protect the vascular repair and minimize additional soft-tissue injury (Connolly, 1970; McNamara et al., 1973).

Nonunion: The literature suggests that 4 months is a reasonable period of time for humeral shaft fractures to unite (Foster et al., 1985; Zuckerman et al., 1993). Nonunion is present when healing is no longer evident. The nonunion rate following humeral shaft fracture ranges from 0-15\% (Naiman et al., 1970) .The proximal and distal thirds of the humerus are at increased risk of nonunion. Other factors associated with nonunion include a transverse fracture pattern, fracture distraction, soft-tissue interposition and inadequate immobilization (Mast et al., 1975; Naiman et al., 1970). Interestingly, higher rates of nonunion have been reported after operative treatment than nonoperative management (Mast et al., 1975). 


\section{MATERIALS AND METHODS}

During the period from Jan 2008 to Jun. 2009 seventy-eight fractures of humeral shaft were treated at Orthopaedic Department in the Tikrit Teaching hospital. 20 fractures considered suitable for the study, those excluded are shown in the Table 1.

There were $13(65 \%)$ male and $7(35 \%)$ female. The age of the patients rang from 17-72 year. A fracture of the humeral shaft was defined as a fracture occurring below the surgical neck and above the epicondyles.

Fractures were divided into those occurring in the upper, middle and lower thirds of the shaft and at the sites of juxtaposition for a total of five groups. In the acutely fractured patient the application method of the ' $U$ ' shaped coaptation slab was standard. The patient was seated on a low stool, leaning to the injured side to expose the axilla. A collar and cuff were applied with elbow at a right angle. The upper arm was wrapped in a single layer of cotton from the shoulder to four inches distal to the elbow. The arm was encased in six inch, eight layers slab that passed from the midclavicular region around the shoulder, down the arm, under the elbow and up the medial aspect of the arm just below the axilla. A wet gauze bandage was used to retain the slab and to mold it to the contours of the arm (Fig. 3). No anesthesia was used and the treatment was on outpatient basis.

All patients were examined the following day; the plaster, position of the limb, circulation and neurologic state were checked and the humeral shaft radiologically examined. Then after two weeks the patient seen and also examined clinically and radiologically, if there is little pain and good alignment and apposition we continue on the coaptation splint and the patient reexamined every two weeks until union evident clinically and radiologically. If there is much pain or any degree of malalignment, we shift to POP cast, which is applied according to these rules:

- The elbow must be in flexion $90^{\circ}$

- The POP cast extends from the mid-palm to the fracture level or not more than one inch above

- The sling must be fixed at the level of the wrist with mid-pronation forearm

- The POP must be light and never be distracting force consist of 4-6 (6 inch) Gypsona wrapped over single layer of cotton

- To correct lateral angulation, the loop should be placed on the dorsum of the wrist, to correct medial angulation, the loop should be placed over the volar side
Table 1: Cases excluded from the study

\begin{tabular}{lc}
\hline Cases & No. of patient \\
\hline Fractures in patient under 17 year & 12 \\
Open fractures & 21 \\
Pathological fractures & 3 \\
Fractures with incomplete & 6 \\
treatment by the department & \\
Incomplete follow up & 9 \\
Insufficient clinical data & 4 \\
Other methods of treatment used & 8 \\
Complicated by nerve injury & 1 \\
Total & 58 \\
\hline
\end{tabular}

- Along sling should be used to correct posterior angulation; short one, to correct anterior angulation

- The arm must be continuously dependent

- Early, active, vigorous, exercises of the longitudinal muscle of the arm (4-6 times daily) are imperative

- Systematic resistant exercise of the fingers and thumb are essential

Then we follow the patient clinically and radiologically every 2-4 week and until the fracture had united and the limb functions were restored. If there was no pain in association with fracture distraction we shift to functional brace which is made in the rehabilitation centre. Then continue our follow up.

Treatment was assessed by the following parameters:

- Alignment: Measurement of humeral angulation in coronal plane (varus and valgus) and in sagittal plane (anterior and posterior) was determined from initial and final radiographs

- Rate of union: Union was assessed clinically; by the absence of bone pain, tenderness and movement on stressing the fracture site. Radiographic union was determined by the evidence of callus formation on plane X-ray. Delayed union was defined as the absence of clinical union 12 weeks after the original trauma

- Limb functions: This was determined by assessing the pain and the return of the movement at the shoulder, elbow and the hand and the final use of the limb and graded as:

- Grade-I: Pain and total restriction preventing all activities

- Grade-II: Less pain and severe restriction preventing or severely impeding daily activities

- Grade-III: Restriction permitting daily activities with some difficulties

- Grade-IV: Minimal restriction not impending daily activities and no pain 
- Grade- V: No restriction of activities and no pain (Hunter, 1982)

\section{RESULTS}

Alignment: All fractures passing to sound union were assessed with respect to the progression of deformity in coronal and sagittal planes by goniometer.

Alignment in coronal plane (Table 2): Five fractures (25\%) were initially undisplaced, 9 fractures $(45 \%)$ had varus angulation and 6 fractures (30\%) had valgus angulation.

At union 7 fractures (35\%) were undisplaced, 10 fractures $(50 \%)$ had varus angulation and 3 fractures (15\%) had valgus angulation.

Alignment in sagittal plane (Table 3): Six fractures $(30 \%)$ were initially undisplaced, 2 fractures $(10 \%)$ had anterior angulation and 12 fractures (60\%) had posterior angulation.

At union 11 fractures (55\%) united without displacement, 1 fracture $(5 \%)$ with anterior angulation and 8 fractures $(40 \%)$ with posterior angulation.

Table 2: Alignment progression in coronal plane

\begin{tabular}{|c|c|c|c|c|c|}
\hline Displacement & $\begin{array}{l}\text { Number of } \\
\text { fractures }\end{array}$ & $\begin{array}{l}\text { Range of } \\
\text { initial } \\
\text { angulation } \\
\text { (degree) }\end{array}$ & Average & $\begin{array}{l}\text { Range } \\
\text { of final } \\
\text { angulation }\end{array}$ & Average \\
\hline \multicolumn{6}{|l|}{ Initially } \\
\hline \multicolumn{6}{|l|}{ Undisplaced } \\
\hline No change & 3 & - & 0 & - & 0 \\
\hline Final varus & 2 & - & 0 & $4-10$ & 7 \\
\hline \multicolumn{6}{|l|}{ Initially varus } \\
\hline Decrease & 7 & May-70 & 31 & $0-28$ & 6 \\
\hline No change & 1 & 11 & 11 & 11 & 11 \\
\hline Increase & 1 & 12 & 12 & 15 & 15 \\
\hline \multicolumn{6}{|c|}{ Initially valgus } \\
\hline Decrease & 3 & May-40 & 19 & $0-10$ & 4 \\
\hline No change & 2 & 9-May & 7 & 9-May & 7 \\
\hline Increase & 0 & 0 & 0 & 0 & 0 \\
\hline VR to VL & 1 & 20-Oct & 15 & $(-5) \_(-7)$ & $(-6)$ \\
\hline
\end{tabular}

Table 3: Alignment progression in sagittal plane

\begin{tabular}{|c|c|c|c|c|c|}
\hline Displacement & $\begin{array}{l}\text { Number of } \\
\text { fractures }\end{array}$ & $\begin{array}{l}\text { Range of } \\
\text { initial } \\
\text { angulation } \\
\text { (degree) }\end{array}$ & Average & $\begin{array}{l}\text { Range } \\
\text { of final } \\
\text { angulation }\end{array}$ & Average \\
\hline \multicolumn{6}{|l|}{ Initially } \\
\hline Undisplaced & & 0 & - & 0 & - \\
\hline No change & 5 & - & - & - & - \\
\hline Final ant. & 0 & 0 & - & 2 & - \\
\hline Final post. & 1 & & & & \\
\hline \multicolumn{6}{|l|}{ Initially ant. } \\
\hline Decrease & 2 & $9-15$ & 12 & $0-10$ & 5 \\
\hline Ant. to post. & 0 & 0 & 0 & 0 & 0 \\
\hline \multicolumn{6}{|l|}{ Initially post } \\
\hline Decrease & 9 & $5-45$ & 13 & $0-10$ & 3 \\
\hline No change & 1 & 5 & - & 5 & - \\
\hline Increase & 2 & $5-15$ & 10 & $7-19$ & 13 \\
\hline
\end{tabular}

Rate of union: (Fig. 6): In our study 19 fractures (95\%) had union with an average time 42 days in males and 44 days in females.

No correlation was found between sex, or type of fracture and the effect of manipulation and the rate of union. One fracture in uncooperative male patient more than 30 year progress to delayed union and the fracture took 13 weeks to get safe union clinically and radiologically. So the incidence of delayed union was $5 \%$.

Function: (Table 4): In assessing the function 12 fractures $(60 \%)$ had grade $\mathrm{V}$ function and 7 fractures (35\%) had grade IV function. Only 1 fracture (5\%) had grade III function especially the shoulder joint and she was an elderly female.

Compensational movement of the upper limb was such that restriction of daily activity was minimal. The average rate of return to full function was 10 weeks and it was fast in patient younger than 35 year and slower and less complete in older.

\section{DISCUSSION}

Good to excellent results can be obtained with nonoperative treatment of patients with humeral shaft fractures. Winfield et al. (1942) and coworkers reported 136 humeral shaft fractures treated with a hanging arm cast; 103 were available for follow-up. There was one delayed union and one nonunion.

\begin{tabular}{lll}
\multicolumn{2}{l}{ Table 4: Show distribution of patients according to functional grade } \\
\hline Grade & No. of Patient & Percentage \\
\hline I & 0 & 0 \\
II & 0 & 0 \\
III & 1 & $5 \%$ \\
IV & 7 & $35 \%$ \\
V & 12 & $60 \%$ \\
\hline
\end{tabular}

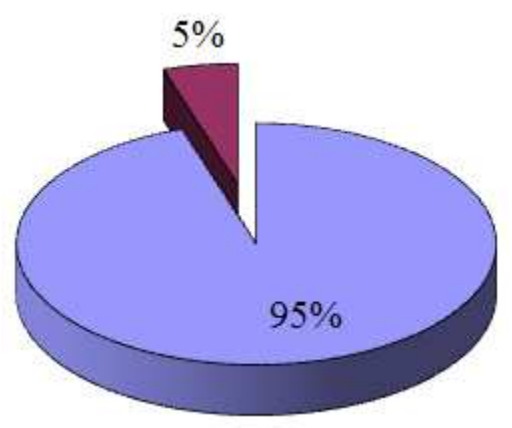

Fig. 6: Pie chart showing distribution according to rate of union 
Hunter (1982) reported 60 humeral shaft fractures treated with a cooptation splint. The arm was suspended by a collar and cuff after application of the splint. Treatment success was based on fracture union, residual deformity and limb function. Fifty-six fractures (93\%) united; all had less than $30^{\circ}$ angulations. The average time to union was 40 days for males and 42 days for females. There was no correlation between healing and patient sex, fracture level, or need for fracture manipulation. With one exception, all patients younger than age 40 recovered full extremity function by 10 weeks. In older patients, functional return was slower. The authors concluded that a coaptation splint could be used effectively to treat patients with humeral shaft fractures.

Balfour et al. (1982) reported 42 patients with a humeral shaft fracture treated with a functional brace. Forty-one fractures $(97 \%)$ united. The time to union averaged 54 days. Varus deformity averaged $9^{\circ}$. Deformity in the anteroposterior plane averaged $6.2^{\circ}$. Thirty-eight patients $(90 \%)$ had full motion of the shoulder and elbow 4 months after fracture.

Our results indicate that the initial deformities of angulation were considerably reduced by our treatment. The U slab and the POP cast act as a dynamic rather than a static splint, correcting angulation to less than $30^{\circ}$ in coronal plane and less than $20^{\circ}$ in sagittal plane.

There was a tendency to residual varus angulation whether the fracture was manipulated or not. The deforming force was sufficient to produce varus angulation from the undisplaced position and in 1 case, to swing a valgus angulation into a varus position. Therefore it did not merely exaggerate the preexisting angulation, but must have resulted from the application and maintenance of the slab and POP cast.

The force producing the posterior displacement could not always be overcome by the conservative treatment, thus resulting in 2 fractures with initial posterior angulation uniting with increased posterior deformity and 1 initially undisplaced fracture unite with posterior angulation.

As 6 of the 12 fractures with initial posterior angulations united without displacement, the deforming force would appear not to be the type of conservative treatment and is most likely to originate from the Triceps muscle and most of the patients feel comfortable with the short sling. To oppose this force would require increasing the weight of the POP cast, which would increase the risk of distraction and consequence nonunion.

Manipulation of the fracture was not required and did affect neither the rate of union nor the final position, as the cast appeared to be capable of correcting angulation deformities.

Perfect anatomical reduction was found not to be essential for satisfactory limb function, which was present with varus angulation and posterior bowing. This supports the findings of Kennermann (1966) who noted good functional results in the presence of residual coronal and sagittal plane angulation, providing the deformity did not exceed $30^{\circ}$.

The incidence of delayed union compares favorably with other reported series, although the definition of delayed union is variable.

The method of assessment of limb function has limitation, but despite that, it is apparent that significant functional impairment was not found. Attempts to define final function by methods used by other reports show similar result.

Regarding open reduction and internal fixation of fracture shaft humerus, Heim et al. (1993) and associates reported 127 patients with humeral shaft fractures also stabilized using plates and screws. Patient age averaged 51 years. Nineteen patients had associated radial nerve palsy; an additional four patients developed palsies after fracture manipulation. Of the 127 patients, 102 were available for follow-up 1 year after fracture. Eighty-nine patients (85\%) had full functional recovery of their upper extremity. Two patients had postoperative radial nerve palsy, four developed a postoperative infection, five had early fixation failure and two developed a nonunion. Stern et al. (1984) and colleagues reported 70 humeral shaft fractures stabilized with several types of intramedullary devices between 1970 and 1981. Complications developed in 47 $(67 \%)$ of the fractures; $45(64 \%)$ required at least one additional operative procedure. Of the 60 fractures that were surgically treated within 6 weeks of injury, nine $(15 \%)$ developed a delayed union and five $(8.3 \%)$ did not unite. Three of 10 fracture $(10 \%)$ that had surgery more than 6 weeks after injury never united despite additional procedures. Delayed union and nonunion were more common in open fractures $(33 \%)$ than in closed fractures (21\%) and after an open nailing (39\%) compared with closed or semi-open nailing (9\%). Adhesive capsulitis of the shoulder developed in 56\% of patients stabilized using an antegrade technique. However, elbow motion was not restricted in patients who were stabilized using a retrograde technique.

So comparing ours and other authors results of conservative treatment with those used ORIF revealed that both gave good functional results but the conservative treatment has much less complication. Therefore we should not operate on fractures of the shaft unless there is clear indication (Table 5). 
Table 5: Indications of ORIF in fractured shaft humerus

Open fracture.

Associated vascular injury.

Floating elbow.

Bilateral humerus fractures.

Humerus fracture in polytrauma patient.

Failure of conservative treatment.

Radial nerve dysfunction after fracture manipulation.

Pathological fracture.

Nonunion.

Unacceptable malunion.

\section{CONCLUSION}

Several features about the humerus cause fractures of that bone to present special attention in treatment make it necessary to depart from common lines of treatment of fractures of long bones.

These features are:

- It is the most freely movable long bone and its movement can be amplified by the movement of the scapula. So it can overcome wide range of malalignment and malrotation

- Its entire function is that of a lever, so that nearly all stress is in tension or at an angle to its long axis. The bone has to stand comparatively little stress in compression

- When at rest while the person is standing, the axis of the bone hangs vertically and is influenced by gravity alone, this can be used effectively for treatment

- It is a single bone, well enclosed in soft tissues (mainly muscles) which give very good vascular supply and can mask malunion in any plane with acceptable cosmetics

- Fractures of bones with a rich blood supply, such as the rib, femur or humerus, where there is slight motion at the fracture site, usually heal rapidly, provided that there is no infection or mechanical interference, such as excessive trauma, soft tissue interposition, or gross malposition

- The acceptable alignment is:

$$
\begin{aligned}
& <20^{\circ} \text { anteroposterior } \\
& <30^{\circ} \text { varus or valgus }
\end{aligned}
$$

With very good functional outcome because of wide range of movement in the upper limb which can be overcome these deformity

From these features we can conclude that: In fracture humerus, like fracture clavicle, neither rigid immobilization nor perfect alignment are of great importance for final outcome of the fracture. So conservative treatment is the most effective way of treatment and the operative treatment can has adverse effect on the outcome in case of bad judgment and should be limited as much as possible to these indications (Table 5).

Recommendations: Because of the high union rate and good to excellent functional outcome we recommend conservative treatment for fracture shaft humerus as treatment of choice and to operate only in the presence of strict indications.

\section{REFERENCES}

Balfour, G.W., V. Mooney and M.E. Ashby, 1982. Diaphyseal fractures of the humerus treated with a ready-made fracture brace. J. Bone Joint Surg., 64A: 11-13. PMID: 7054192.

Chapman, M.W., 2003. Fractures and Dislocations of the Shoulder Girdle and Humerus. In: Chapman's Orthopedic Surgery. JB Lippincott, Philadelphia, pp: 1004-1012. ISBN: 13: 9780781714877

Christensen, S., 1967. Humeral shaft fractures: Operative and conservative treatment. Acta Chir. Scand., 133: 455-460. PMID: 6073784

Connolly, J., 1970. Management of fractures associated with arterial injuries. Am. J. Surg., 120(3): 331. PMID: 5456914

Epps, C.H., Jr. and R.E. Grant, 1991. Fractures of the Shaft of the Humerus. In: Rockwood and Green's Fractures in Adults. JB Lippincott, Philadelphia, pp: 843-869. ISBN: 0-397-50975-8

Foster, R.J., G.L. Dixon and A.W. Bach, 1985. Internal fixation of fractures and non-unions of the humeral shaft: Indications and results in a multi-center study. J. Bone Joint Surg., 67A: 857-864. PMID: 4019533

Heim, D., F. Herkert and P. Hess., 1993. Surgical treatment of humeral shaft fractures-the Basel experience. J. Trauma., 35: 226-232. journals.lww.com/jtrauma/toc/1993/08000

Hunter, S.G., 1982. The closed treatment of fractures of the humeral shaft. Clin. Orthop., 164: 192-198. PMID: 7067285

Kenermann, L., 1966. Fractures of the humeral shaft. J. Bone Joint Surg., 48B: 105-111. PMID: 5909054

Mast, J.W., P.G. Spiegel and J.P. Harvey, 1975. Fractures of the humeral shaft: A retrospective study of 240 adult fractures. Clin. Orthop., 112: 254-262. PMID: 1192642

McNamara, J.J., D.K. Brief and J.F. Stremple 1973. Management of fractures with associated arterial injury in combat casualties. J. Trauma, 13: 17-19. PMID: 4687242 
Naiman, P.T., A.J. Schein and R.S. Siffert, 1970. Use of ASIF compression plates in selected shaft fractures of the upper extremity: A preliminary report. Clin. Orthop., 71: 208-209. PMID: 5433382

Naver, L. and J.R. Aalberg, 1986. Humeral shaft fractures treated with a ready-made fracture brace. Arch. Orthop. Trauma Surg., 106: 20-22. DOI: 10.1007/BF00435647

Pollock, F.H., D. Drake and E.G. Bovill, 1981. Treatment of radial neuropathy associated with fractures of the humerus. J. Bone Joint Surg., 63A: 239-243. PMID: 7462281

Sarmiento, A., P.B. LaFerte and E.G. Galvin. 1977. Functional bracing of fractures of the shaft of the humerus. J. Bone Joint Surg., 59A: 596-601. PMID: 873955

Stern, P.J., D.A. Mattingly and D.L. Pomery et al., 1984. Intramedullary fixation of humeral shaft fractures. J. Bone Joint Surg., 66A: 639-646. PMID: 6725312
Stewart, M.J. and J.M. Hundley, 1955. Fractures of the humerus: A comparative study in methods of treatment. J. Bone Joint Surg., 37A: 681-692. PMID: 13242602

Terry Canal, S., 2008. Fractured Shaft Humerus. In: Campbell's Operative Orthopaedic. 11th Edn. 3389-3400. Mosby Elsevier. Philadelphia. ISBN: 978-0-323-03329-9

Ward, E.F., F.H. Savoie and J.L. Hughes, 1992. Fractures of the Diaphyseal Humerus. In: Skeletal Trauma, Browner, B.D., J.B. Jupiter, A.M. Levine and P.G. Trafton (Eds.). WB Saunders, Philadelphia, pp: 1177-1200. ISBN: 0-7216-2726-9

Zuckerman, J.D., C. Giordano and H. Rosen, 1993. Humeral Shaft Nonunions. In: Complications of Shoulder Surgery, Bigliani, L.U. (Ed.). Williams and Wilkins, Baltimore, pp: 173-189. ISBN: 0683007513 